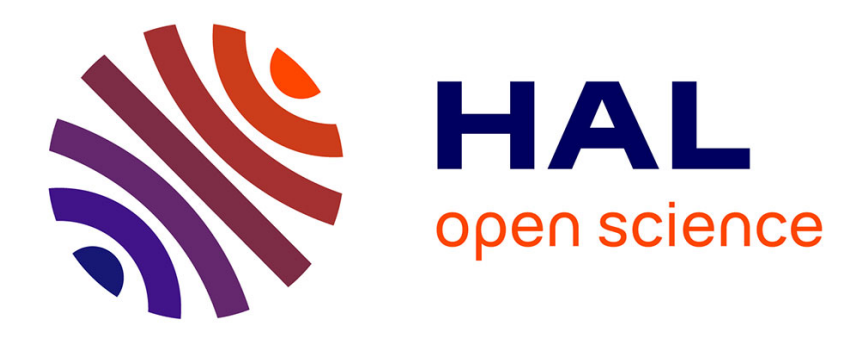

\title{
Analysis and Improvement of mobility procedures for mobile relays in LTE networks \\ Yangyang Chen, Xavier Lagrange
}

\section{To cite this version:}

Yangyang Chen, Xavier Lagrange. Analysis and Improvement of mobility procedures for mobile relays in LTE networks. 26th IEEE International Symposium on Personal, Indoor and Mobile Radio Communications (PIMRC 2015) , Aug 2015, Hongkong, China. pp.1769-1774, 10.1109/PIMRC.2015.7343585 . hal-01308352

\section{HAL Id: hal-01308352 \\ https://hal.science/hal-01308352}

Submitted on 27 Apr 2016

HAL is a multi-disciplinary open access archive for the deposit and dissemination of scientific research documents, whether they are published or not. The documents may come from teaching and research institutions in France or abroad, or from public or private research centers.
L'archive ouverte pluridisciplinaire HAL, est destinée au dépôt et à la diffusion de documents scientifiques de niveau recherche, publiés ou non, émanant des établissements d'enseignement et de recherche français ou étrangers, des laboratoires publics ou privés. 


\title{
Analysis and Improvement of mobility procedures for mobile relays in LTE networks
}

\author{
Yangyang Chen, Xavier Lagrange \\ Institut Mines Telecom / TELECOM Bretagne \\ IRISA, Rennes, France \\ Email: $\{$ yangyang.chen, xavier.lagrange@telecom-bretagne.eu\}
}

\begin{abstract}
With the popularity of smart phones and tablets, people make intensive use of these devices on public transport. It is thus interesting to deploy mobile relays in public transportation with the aim of offering high-quality services. In LTE Rel 10 fixed relays are specified. Although mobile relays have been considered by 3GPP, no protocol stack is specified yet. In this paper, we propose to keep the same protocol stack as for fixed relays but introduce the concept of global tunnel, which gathers several tunnels. We analyze the main signaling procedures and show that the proposed solution dramatically reduces the number of messages that each network node should process.
\end{abstract}

Index Terms-Mobile Relay; Global Tunnel; Signaling Procedures; LTE

\section{INTRODUCTION}

Relay nodes can extend cell-edge coverage, increase capacity and provide flexible deployment. They have been extensively studied as a key component of future heterogeneous networks [1]. Relays can be characterized into two categories: fixed relays and mobile relays. Fixed relays are usually deployed at selective locations, such as shaded areas to provide coverage or hot spots to increase the capacity [2]. Fixed relays have already been standardized in release 10 of 3GPP LTE standards.

Currently, with the popularity of smart phones and tablets, people make intensive use of these devices when they are on public transport. When these users are grouped on a bus or a tram, this can be seen as a mobile hot spot. With the aim of offering high-quality services to these users, deploying mobile relay on public transportations is a natural idea. In this context the 3GPP is working on enhancing mobile relay functionality on public transport such as buses, trams, or trains [3].

By mounting a mobile relay in a moving vehicle, wireless connectivity is provided to vehicular UE (User Equipment) in vehicles through the in-vehicle antenna of mobile relay. Wireless connection is provided to mobile relay through the outdoor antenna of mobile relay and the DeNB (Donor eNodeB). By using two separate transmission links, the penetration loss can be eliminated. Some studies have been done on mobile relays. In [4]-[7], the performance of deploying mobile relays in buses is evaluated through Monte-Carlo simulations and stochastic geometry.

When relay-node mobility is considered, multiple path switches of vehicular UEs would be triggered in case of handover if no enhancement is used [8]. In [9], [10], the authors proposed to use the predictability of the movement of high speed trains in order to optimize handover. In [11], the authors discussed several architecture alternatives for mobile relays and proposed optimized mobile relay handover procedures based on mobility area. Mobility area is a set of eNodeBs. Path switches for vehicular UEs are only performed when mobility area changes. Handover latencies of the procedures are analyzed. However, this proposal requires extra planning effort on the operator side and some upgrades of the standard 3GPP based protocols. Until now, no specification is defined for relay node mobility. Several proposals are studied by $3 \mathrm{GPP}$ in [12]. Some consider restrictive assumptions (SGW collocated with eNodeB).

In this paper, we study the different signaling procedures when the relay is embedded in a public transport vehicle and when the architecture for fixed relays is kept. For the sake of simplicity, we consider the case of deploying mobile relays on public buses, but the proposal can be applied to any transport systems: metros, tramways and trains. The sections are organized as follows. Firstly, the main procedures with fixed relay node are described in Section II. In Section III, we propose the concept of global tunnel. In Section IV, we analyze the processing cost on different network nodes in different modes. Numerical results are presented and analyzed in Section V. A conclusion is drawn in Section VI.

\section{MAIN PROCEDURES WITH A FIXED RELAY}

In this section, we analyze the signaling for a typical scenario: a user goes under the coverage of a relay, then connects his/her terminal through the relay to the network, uses his/her terminal (data exchange) and finally leaves the relay coverage. We assume the relay is already attached to the eNodeB (see [13] for more details on the attachment).

\section{A. Principles of the relay architecture}

In the specification [14] the architecture "Proxy S1/X2" is defined for fixed relay node, as shown in Figure 1. The basic idea is to add "home eNB GW" functions into the DeNB in order to provide the SGW/PGW-like functions needed for $\mathrm{RN}$ (Relay Node). The DeNB appears as a MME (for S1), an eNB (for X2) and a SGW to the relay node. Both U-plane and Cplane of the $\mathrm{S} 1$ interface is terminated at the $\mathrm{RN}$. There is only one radio connection between each RN and the DeNB, but there are as many connections as the number of UEs between 
the DeNB and the MME(UE), and between the DeNB and SGW(UE), as shown in Figure 2.

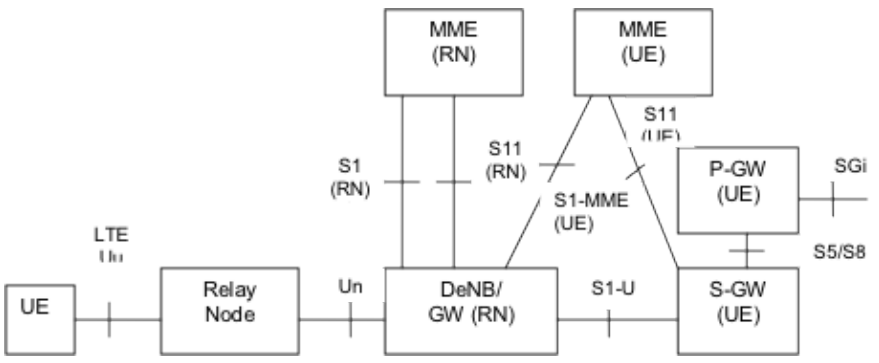

Fig. 1. Relay Architecture (source [14])

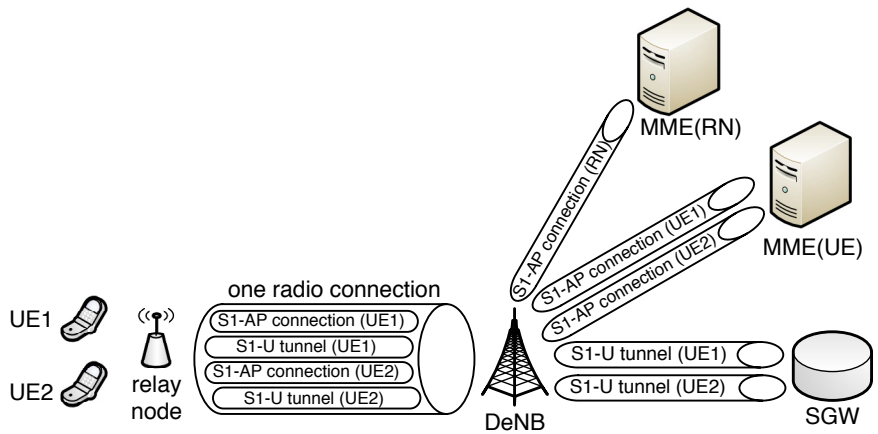

Fig. 2. Tunnels and connections with fixed relays

\section{B. Arrival of a UE in the coverage of a relay}

We consider a UE in IDLE state when no NAS signaling connection between UE and network exists. In IDLE state, the location of the UE is only known by the network at the Tracking Area List (TAL) level. When an inactive UE arrives in the coverage of a relay, depending on whether the relay node has a specific TAI (Tracking Area Identity) or not, the UE may trigger a tracking area update.

1) The relay node has the same TAI as the DeNB: UE activities in IDLE state are restricted to periodic paging reception and serving cell monitoring. In this case, the network is not aware that the UE moves into the coverage of a relay. On receipt of a paging message, the UE performs a service request procedure which results in moving the UE to CONNECTED state. Only at that time the network detects that the UE is in the bus.

2) The relay node has a different TAI from the DeNB: When a UE moves to the coverage of a relay, it detects that it has entered a new TA that is not in the list of TAIs. The Tracking Area Update (TAU) procedure is thus initiated by the UE. The TAU procedure is shown in Figure 3.

In Figure 3 and following ones, we show the number of messages processed by each entity, which is denoted by $M_{y}^{x}$ for procedure $y$ (e.g. $y=\mathrm{TAU}$ ) and entity $x$ (e.g. $x=\mathrm{MME}$ ). This is used latter for the analytical evaluation.

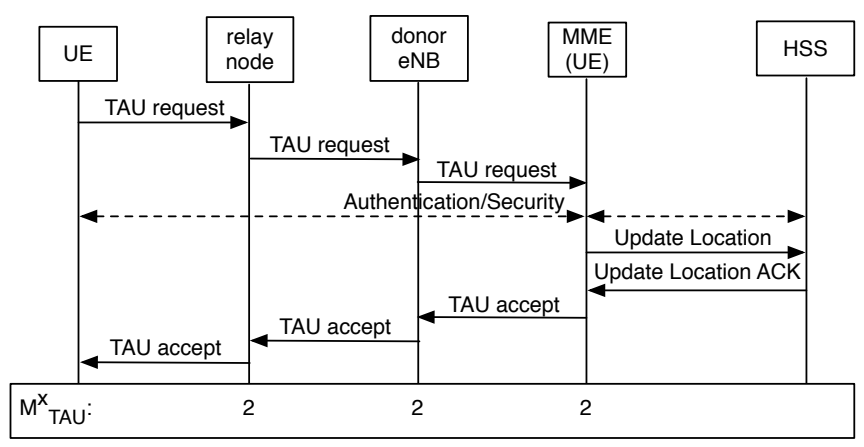

Fig. 3. Tracking Area Update Procedure (TAU)

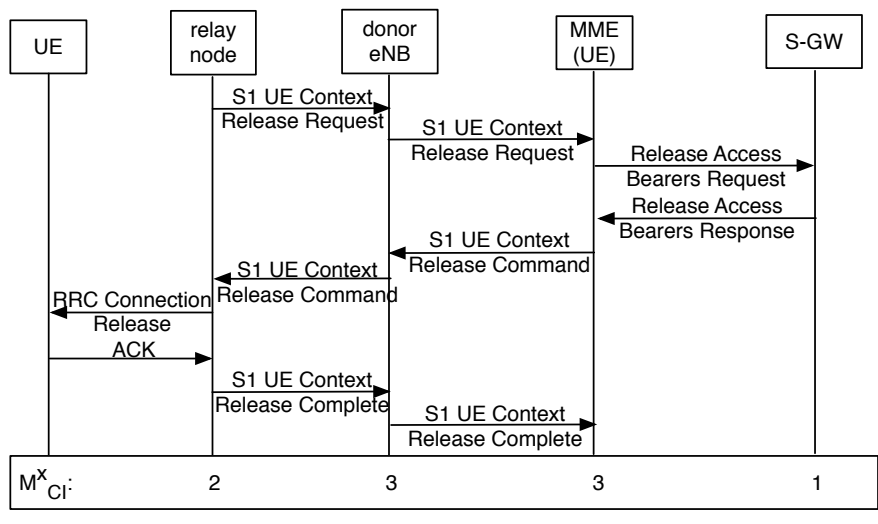

Fig. 4. Switch from CONNECTED state to IDLE state (CI)

\section{Switch from CONNECTED state to IDLE state}

A UE can be either in CONNECTED state or in IDLE state. If a UE does not transmit or receive any data for a while, the eNB releases the RRC (Radio Resource Control) connection and asks the MME to release the S1 tunnel and the S1-AP connection. That event is triggered by the eNB, which manages a RRC inactivity timer. The UE then switches from CONNECTED state to IDLE state, as shown in Figure 4. Typically, the RRC inactivity timer is in the range of a few seconds to a few tens of seconds.

\section{UE triggered Service Request procedure}

When there is uplink data to be sent from a UE in IDLE state to the network, the UE performs Service Request procedure illustrated in Figure 5. A connection is setup in the control plane and user plane, allowing the UE to receive and send data traffic.

\section{E. Network triggered Service Request procedure}

When the network has downlink traffic to deliver to a UE in IDLE state, the Network triggered Service Request procedure is performed, as shown in Figure 6. First the SGW buffers the downlink data and identifies which MME is serving the UE, then the SGW asks the MME to trigger the paging procedure in order to find and activate the UE. If the relay node has a same TAI as the DeNB, the UE is paged in all eNBs belonging to the TAL (Tracking Area List). If the relay node has a specific 


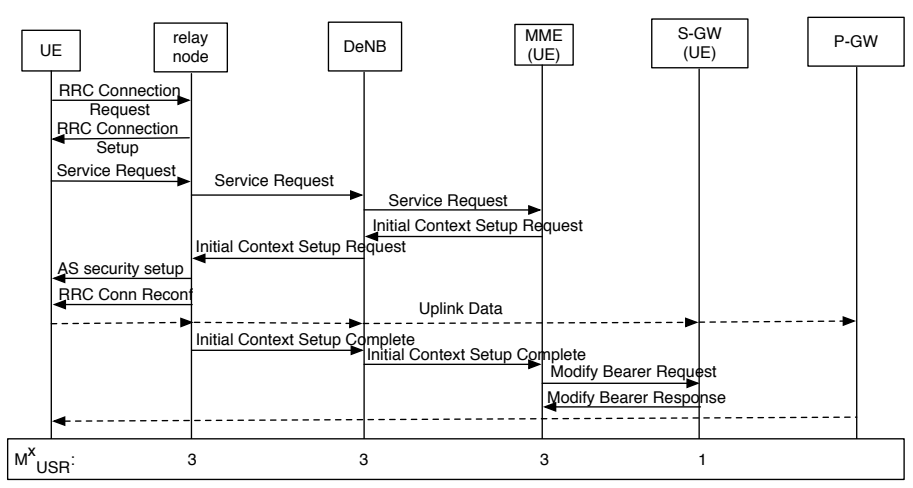

Fig. 5. UE triggered Service Request procedure (USR)

TAI, the paging message is only sent to the relay node. Once the UE is located, it triggers the Service Request procedure described above.

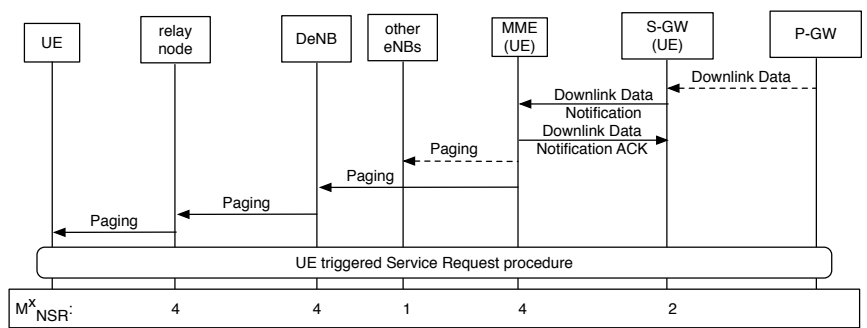

Fig. 6. Network triggered Service Request procedure (NSR)

\section{The PRoposed Scheme}

In this section, we first propose the concept of global tunnel, and we explain how it can be used for mobile relays. We introduce two options: X2 global tunnel and S1 global tunnel.

\section{A. The Concept of Global Tunnel}

In order to reduce the number of signaling messages in mobile relay scenario, we propose the concept of global tunnel. The idea is to gather several tunnels in the same global tunnel and use this global tunnel to transmit the data traffic for vehicular UEs served by the same mobile relay. The global tunnel uses the GTP-U protocol. A first simple idea is to encapsulate GTP-U data units related to UEs into GTP-U data units related to the global tunnel. However, this would increase the number of protocols in the stack. We thus propose a simpler solution. According to the specification [15], the GTP-U header includes an extension field named Extension Header Flag (E). We propose to use this option to transport a second TEID (Tunnel endpoint identifier) in the extension header that identifies the tunnel that is specific to a UE. The TEID that is in the standard header identifies the global tunnel. The extended GTP-U header is shown in Figure 7. Note that intermediate nodes do not need to be modified to transport global tunnels.

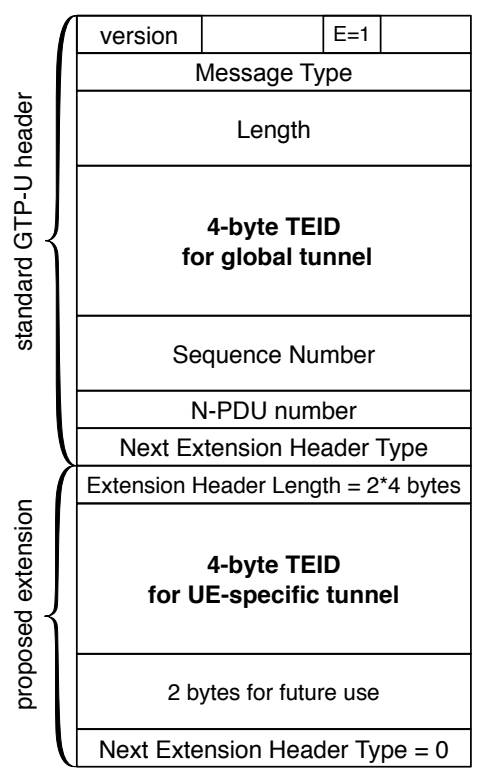

Fig. 7. Extended GTP-U header

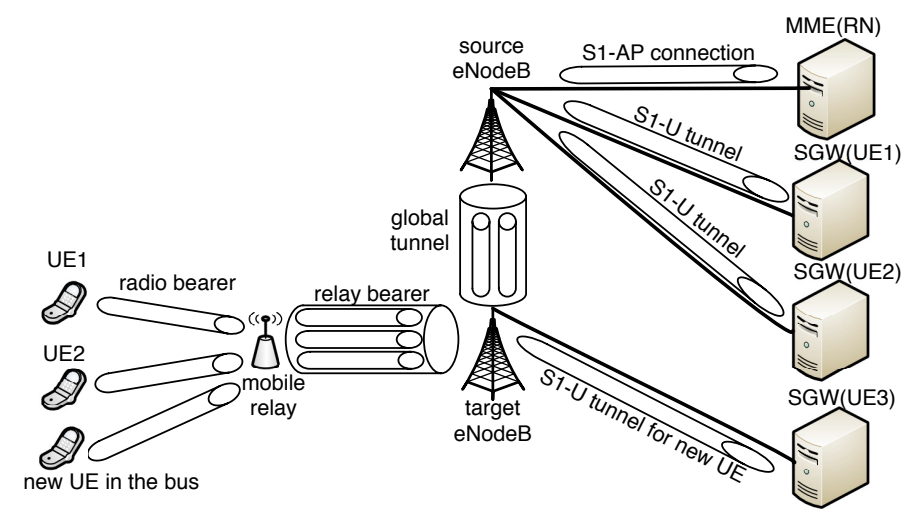

Fig. 8. The global X2 tunnel during the handover of mobile relay

\section{B. Global X2 Tunnel}

During the handover of mobile relay, we propose to set up a global tunnel between the source DeNB and the target DeNB for the data forwarding of vehicular UEs, as shown in Figure 8. All vehicular UEs that are connected to the mobile relay before the handover have their data transmitted on the following path: mobile relay / target DeNB / source DeNB / SGW (UE). But for UEs that connects through the mobile relay after the handover, a direct path is established between the target DeNB and SGW(UE). For a given UE, its mobility anchor is the serving DeNB when it attaches to the mobile relay. The global X2 tunnels are kept as long as it's necessary. They are released as soon as all UEs that use it leave the bus.

With the global X2 tunnel, mobile relay handover procedure is shown in Figure 9. Handover preparation and execution phases are basically the same as specified in [12], except the source DeNB includes the global tunnel information within the Handover Request message. The target DeNB establishes the global tunnel and includes the information within the 
Handover Request Acknowledge message to indicate that it accepts the proposed forwarding of downlink data for this bearer. The global tunnel between source DeNB and target DeNB is established in handover preparation phase.

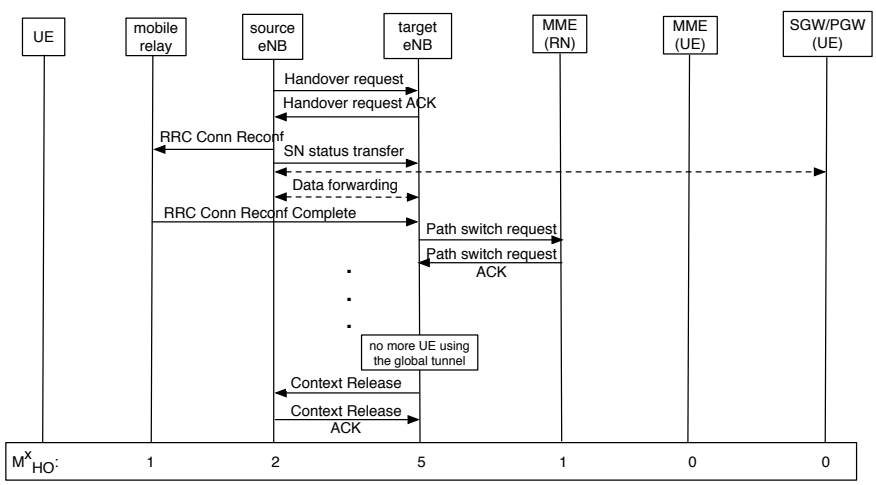

Fig. 9. Mobile relay handover procedure based on global X2 tunnel (HO)

In the handover completion phase, path switch is triggered by the target DeNB. The target DeNB sends the Path Switch Request message to the MME (RN) to inform it that new tunnels and connections for vehicular UEs should be set through the target DeNB. Data on all existing connections are routed through the $\mathrm{X} 2$ interfaces. When all UEs have left the bus, a Context Release message is then sent to the source DeNB by the target DeNB. The source DeNB releases data and control plane related resources.

\section{Global S1 Tunnel}

In the following we assume that mobile relay and vehicular UEs are served by the same MME. This is not restrictive because the number of MMEs in a network is generally low. Instead of having as many S1-U tunnels as the number of vehicular UEs between the DeNB and the SGW (UE), global S1 tunnel can be established between the DeNB and the SGW (UE), as illustrated in Figure 10.

The mobile relay handover procedure based on global S1 tunnel is shown in Figure 11. It is basically the same as the standard handover procedure. In the handover completion phase, the target DeNB sends a Path Switch Request message to the MME (RN\&UE) to inform it that the mobile relay has changed the cell, the global tunnel information are included in this message. After receiving this message, the MME (RN\&UE) asks the SGW (UE) to switch the downlink data path. Then the MME sends back Path Switch Request Acknowledge to the target DeNB. The global S1 tunnel is thus established between the target DeNB and the SGW (UE) in this phrase.

\section{Discussions on the RRC inactivity timer}

The interactivity of applications in smart phones generates frequent switching of UEs between IDLE and CONNECTED states in the standard case. A lot of signaling messages (i.e. service request procedures and resource release procedure) are thus exchanged between the network entities (DeNB, MME,

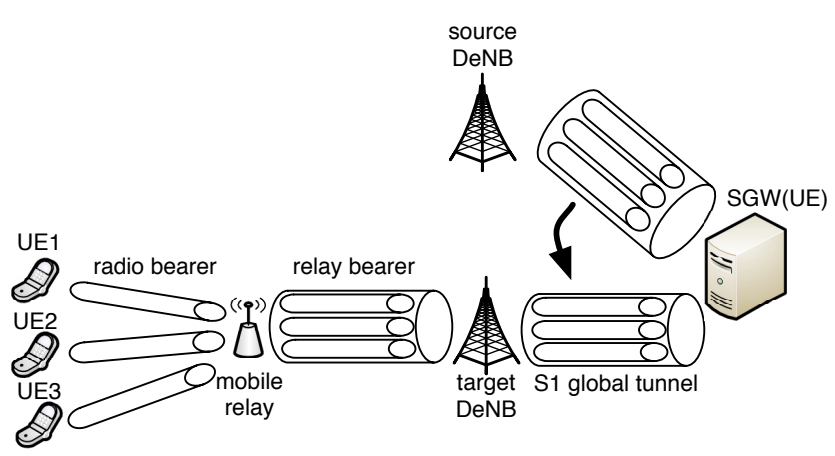

Fig. 10. The global S1 tunnel during the handover of mobile relay

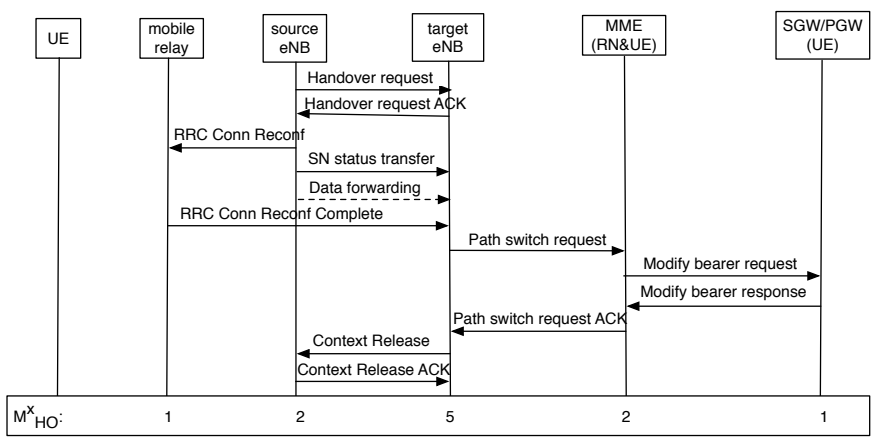

Fig. 11. Mobile relay handover procedure based on global S1 tunnel (HO)

SGW). In mobile relay scenario the mobility of users is constrained by the public transport (buses in our case): when a UE is connected to a RN, this is valid for typically ten minutes or more. Based on this characteristic, when a vehicular UE is inactive, it's interesting to keep its S1 tunnels active in order to have a very fast Service Request procedure, so that the related signaling messages can be reduced. This is possible if the network is aware of the location of the vehicular UE, or in other words, if a specific TAI is defined for each mobile relay.

Therefore, we propose to keep the RRC inactivity timer on the radio interface in order to spare radio resource, but to deactivate it for the tunnels between the RN and the DeNB, and between the DeNB and both the MME and the SGW. Note that this requires maintaining the RRC connection between the $\mathrm{RN}$ and the DeNB. If there is absolutely no traffic in the bus, some "keep alive" messages should be transmitted by the RN. However, if there are some vehicular UEs, the probability of such an event is low. Defining a specific TAI for a mobile relay is thus very interesting.

\section{Processing Cost Evaluation}

In this section, we provide a simple analysis based on the computation of the average processing cost of signaling messages. The processing cost is defined as the number of requests received and processed by an entity per unit time. We consider a hexagonal network with cell radius $r$ and all cells in the same tracking area. There are on average $N$ users in a bus. Users get in the bus and stay in it for $\bar{T}_{D}$ on average. 
They use applications that generate sporadic traffic. Hence, the UE switches between ECM_Idle and ECM_Connected states. Let $\bar{T}_{C}$ and $\bar{T}_{I}$ be the average duration of CONNECTED and IDLE states respectively. The activity of the application is triggered by the terminal with probability $\beta$ (and by the network with probability $1-\beta$ ). Assume that all UEs are in IDLE state when they get on the bus. The parameters are listed in the Table I.

TABLE I

DEFINITION AND DEFAUlT VALUES FOR THE PARAMETERS

\begin{tabular}{|c|c|c|}
\hline Definition & Variables & Default value \\
\hline Average number of UEs in a bus & $N$ & 10 \\
\hline Average dwell time of a UE in a bus & $\bar{T}_{D}$ & $600 \mathrm{~s}$ \\
\hline Probability of UE-triggered service request & $\beta$ & $50 \%$ \\
\hline Average time in ECM_Connected state & $\bar{T}_{C}$ & $10 \mathrm{~s}$ \\
\hline Average time in ECM_Idle state & $\bar{T}_{I}$ & $80 \mathrm{~s}$ \\
\hline Average speed of a bus & $\nu$ & $4 \mathrm{~m} / \mathrm{s}$ \\
\hline Radius of the hexagonal cell & $r$ & $700 \mathrm{~m}$ \\
\hline
\end{tabular}

The probability that the UE is in CONNECTED state is $\chi=\frac{\bar{T}_{C}}{\bar{T}_{1}+\bar{T}_{C}}$. The average number of transitions from CONNECTED state to IDLE state or from IDLE state to CONNECTED state per unit time is $\frac{1}{\bar{T}_{I}+\bar{T}_{C}}$. In order to compute the number of messages due to handover events, we use the fluid-flow model [16] [17] to obtain the average number of cell change with perimeter length $L$. Let $\nu$ and $S$ be the average speed of a bus and the area of the region respectively. So the average number of cell change $\alpha=\frac{\nu L}{\pi S}=\frac{4 \sqrt{3}}{3 \pi} \frac{\nu}{r}$ for an hexagonal network. The average number of handover per UE per unit time is $\alpha \chi$, the average number of cell change in IDLE state is $\alpha(1-\chi)$.

Next we compute the processing cost and compare the total processing cost in direct mode where UEs in a bus directly connect to eNodeB and the processing cost in mobile relay mode. In mobile relay mode, we consider two cases: mobile relay with the same TAI as the DeNB and mobile relay with a specific TAI.

\section{A. Direct Mode}

There is no tracking area update procedure in direct mode, but there are service request procedure, resource release procedure and as many handovers as the number of UEs in CONNECTED state during each cell change. The total number of incoming messages $C_{\mathrm{DM}}^{x}$ processed by entity $x$ per bus per unit time in direct mode is:

$$
C_{\mathrm{DM}}^{x}=N\left[\frac{\beta M_{\mathrm{USR}}^{x}+(1-\beta) M_{\mathrm{NSR}}^{x}+M_{\mathrm{CI}}^{x}}{\bar{T}_{I}+\bar{T}_{C}}+\alpha \chi M_{\mathrm{HO}}^{x}\right]
$$

\section{B. Mobile Relay Mode}

1) same TAI: There is no tracking area update procedure in this relay mode, but there are service request procedure, resource release procedure and one grouped handover of relay node during each cell change. The total number of incoming messages $C_{\mathrm{R} 1}^{x}$ processed by entity $x$ per bus per unit time in this mode is:

$$
C_{\mathrm{R} 1}^{x}=N \frac{\beta M_{\mathrm{USR}}^{x}+(1-\beta) M_{\mathrm{NSR}}^{x}+M_{\mathrm{CI}}^{x}}{\bar{T}_{I}+\bar{T}_{C}}+\alpha M_{\mathrm{HO}}^{x}
$$

2) specific TAI: In order to keep the $S 1$ tunnels active and have a very fast Service Request procedure, a specific TAI is defined for mobile relay. So in this case, there are no service request procedure and resource release procedure, but tracking area procedure is triggered when a UE gets in the bus and gets off the bus. The total number of incoming messages $C_{\mathrm{R} 2}^{x}$ processed by entity $x$ per bus per unit time in this mode is:

$$
C_{\mathrm{R} 2}^{x}=N \frac{2}{\bar{T}_{D}} M_{\mathrm{TAU}}^{x}+\alpha M_{\mathrm{HO}}^{x}
$$

\section{NumericAl RESUlts AND ANALYSis}

In this section, we compute the processing cost based on real statistics in Rennes, France (the average dwell time of a UE in a bus and the average speed of a bus). The statistics are presented in Table I. We mainly consider the processing cost on the core network side (eNodeB, MME, SGW), as RN operates on radio interface and appears as a UE for DeNB, the processing cost on $\mathrm{RN}$ is not included.

\section{A. Impact of the average number of UEs in a bus}

First we evaluate the processing cost against the average number of UEs, as shown in Figure 12. Different modes (direct mode, mobile relay mode $\mathrm{R} 1$ with the same TAI and mobile relay mode $\mathrm{R} 2$ with a specific TAI ) are presented from left to right in the figure. As the number of UEs in a bus increases, the number of signaling messages also increases in all modes. Mode R1 does not reduce any signaling messages compared with direct mode. This is because the handover is not frequent as the bus moves relatively slowly, the advantage of grouped handover in relay mode is not obvious.

However, in mobile relay mode R2 the number of signaling messages is dramatically reduced about $85 \%$ compared with direct mode when there are 20 UEs on average in a bus. This is due to the combination of global tunnels with a specific tracking area and the maintenance of S1 tunnels for inactive vehicular UEs, the signaling messages for service request procedure and resource release procedure are saved.

\section{B. Impact of the average time in ECM_Connected state and ECM_Idle state}

Then we study the impact of the average time in ECM_Connected state and ECM_Idle state, as shown in Figure 13. We consider different applications, such as web browsing, social networks (i.e Facebook, tweeter). The average time in CONNECTED state is set to $[10,30,60]$, the average time in IDLE state is set to $[80,160]$. The average number of UEs in a bus is set to 10 .

As we can see, the number of signaling messages in mobile relay mode $\mathrm{R} 2$ is dramatically reduced and is independent of the type of applications. This is because no matter whatever the application behavior is, the $\mathrm{S} 1$ tunnels are kept alive between the RN and the DeNB, and between the DeNB and both the MME and the SGW. 


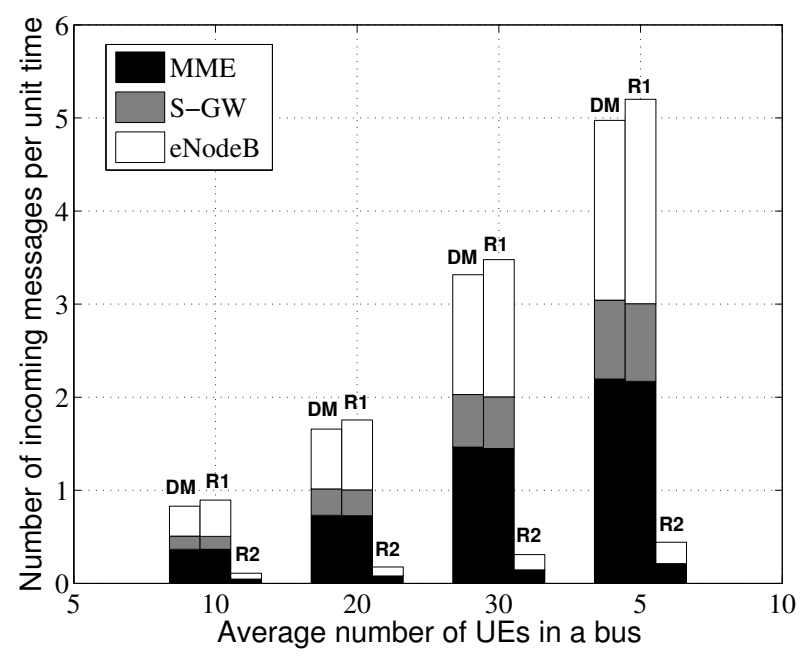

Fig. 12. Processing cost in terms of the average number of UEs in a bus

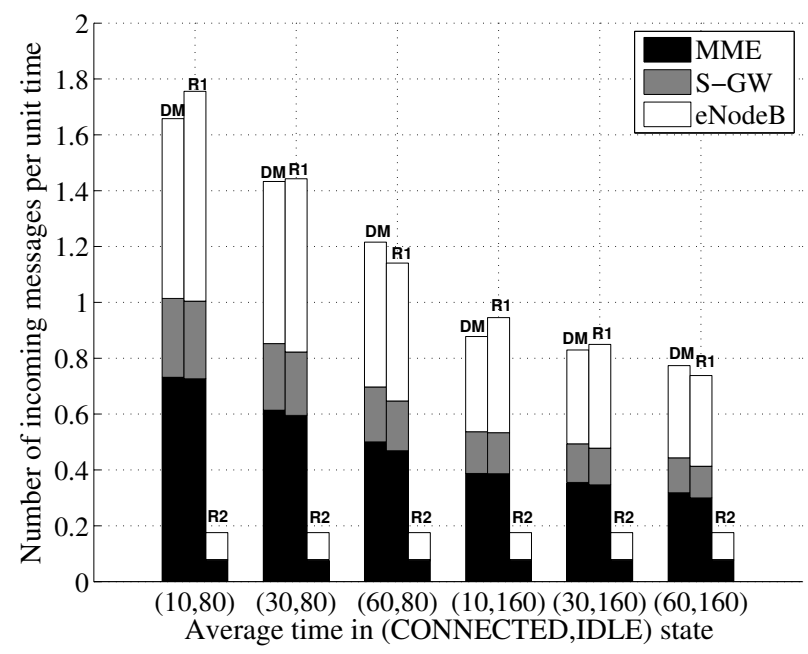

Fig. 13. Impact of the average time in ECM_Connected state and ECM_Idle state

\section{CONCLUSION}

In this paper, we studied an architecture with mobile relays. We proposed to keep the same protocol stack that was defined for fixed relay, and to extend it to mobile relays. We proposed the concept of global tunnels which is compatible with the GTP protocol. Without adding any protocol in the stack, it's possible to group several handovers while keeping the possibility of managing the tunnel of each UE individually. We showed that the number of signaling messages that each network node should process can be dramatically reduced when global tunnels are combined with a specific tracking area and when S1 tunnels are kept alive for inactive vehicular UEs. The solution introduced in this paper can be easily applied to any transport systems: metros, tramways and trains. Therefore, in a future work, we will extend the analysis with the consideration of different types of applications and different scenarios and evaluate the performance by simulations.

\section{ACKNOWLEDGMENT}

This work was performed within project SYSTUF, which is subsidized by the french ministry of Industry in the framework of the AMI ITS program.

\section{REFERENCES}

[1] A. Damnjanovic, J. Montojo, Y. Wei, T. Ji, T. Luo, M. Vajapeyam, T. Y. Song, and D. Malladi, "A survey on 3gpp heterogeneous networks," IEEE Wireless Communications, vol. 18, no. 3, pp. 10-21, June 2011.

[2] M. Minelli, M. Ma, M. Coupechoux, J.-M. Kelif, M. Sigelle, and P. Godlewski, "Optimal relay placement in cellular networks," IEEE Transactions on Wireless Communications, vol. 13, no. 2, pp. 998-1009, February 2014.

[3] 3GPP, Overview of 3GPP Release 12, V0.0.5, September 2012.

[4] Y. Sui, A. Papadogiannis, and T. Svensson, "The potential of moving relays - a performance analysis," in 2012 IEEE 75th Vehicular Technology Conference (VTC Spring), May 2012, pp. 1-5.

[5] Y. Sui, J. Vihriala, A. Papadogiannis, M. Sternad, W. Yang, and T. Svensson, "Moving cells: A promising solution to boost performance for vehicular users," IEEE Communications Magazine, vol. 51, no. 6, pp. 62-68, June 2013.

[6] Y. Chen and X. Lagrange, "Downlink capacity gain analysis of mobile relay in lte-advanced network," in 2014 IEEE 11th Consumer Communications and Networking Conference (CCNC), January 2014, pp. 544-550.

[7] Y. Chen, P. Martins, L. Decreusefond, X. Lagrange, and F. Yan, "Stochastic analysis of a cellular network with mobile relays," in GLOBECOM 2014 : IEEE Global communications conference, 2014.

[8] L. Chen, Y. Huang, F. Xie, Y. Gao, L. Chu, H. He, Y. Li, F. Liang, and Y. Yuan, "Mobile relay in lte-advanced systems," IEEE Communications Magazine, vol. 51, no. 11, pp. 144-151, November 2013.

[9] M.-S. Pan, T.-M. Lin, and W.-T. Chen, "An enhanced handover scheme for mobile relays in lte-a high-speed rail networks," IEEE Transactions on Vehicular Technology, vol. PP, no. 99, p. 1, 2014.

[10] Q. Huang, J. Zhou, C. Tao, S. Yi, and M. Lei, "Mobile relay based fast handover scheme in high-speed mobile environment," in IEEE Vehicular Technology Conference (VTC Fall), September 2012, pp. 1-6.

[11] C. Pietsch, S. Brueck, M. Farber, T. Moraes, M. Khanfouci, J. Vihriala, M. D. Nisar, E. Seidel, T. Svensson, A. Papadogiannis, Y. Sui, and M. Sternad, "Moving Relays and Mobility aspects," Artist4G deliverable, Tech. Rep., May 2012.

[12] 3GPP TR 36.836, Evolved Universal Terrestrial Radio Access (EUTRA); Study on mobile relay, Release 12, June 2014.

[13] Y. Chen and X. Lagrange, "Architecture and Protocols of EPC-LTE with relay,” RSM - Dépt. Réseaux, Sécurité et Multimédia (Institut MinesTélécom-Télécom Bretagne), Tech. Rep., 2013.

[14] 3GPP TS 36.300, Evolved Universal Terrestrial Radio Access (E-UTRA) and Evolved Universal Terrestrial Radio Access Network (E-UTRAN); Overall description; Stage 2, Release 10, Febuary 2015.

[15] 3GPP TS 29.281, General Packet Radio System (GPRS) Tunnelling Protocol User Plane (GTPv1-U), Release 12, January 2015.

[16] R. Thomas, H. Gilbert, and G. Mazziotto, "Influence of the moving of the mobile stations on the performance of a radio mobile cellular network," Proceedings of the Nordic Seminar on Digital Land Mobile Radio Communications, 1988.

[17] T. X. Brown and S. Mohan, "Mobility management for personal communications systems," IEEE Transactions on Vehicular Technology, vol. 46, no. 2, pp. 269-278, May 1997. 